\title{
Conformación de una identidad docente entre profesionistas universitarios contratados por asignatura en el nivel medio superior
}

\author{
Conformation of a teaching identity between university \\ professionals hired by course at the higher middle level
}

Margarita Martínez Martínez*

MARÍA DE IBARROLA**

Este artículo muestra los resultados de una investigación cualitativa sobre la formación como docentes de doce profesionistas contratados como profesores de asignatura en el Colegio de Bachilleres de la Ciudad de México. Con base en entrevistas cualitativas, examina tres transiciones profesionales que han vivido estos profesores conforme a la propuesta base de investigadores de la Universidad de Sherbrooke, quienes son expertos en la teoría de las transiciones. Esta teoría sirvió como eje para construir tres categorías de análisis: de una pedagogía de la intuición a una pedagogía sistemática; del saber sobre una disciplina al saber cómo enseñarla; y de una identidad como profesionista de diversas disciplinas a una identidad docente. El desarrollo de estas transiciones se analiza a partir de la recuperación de la trayectoria de los entrevistados en el Colegio de Bachilleres en tres momentos clave: el ingreso como profesor novel, el momento de la entrevista como profesor más experto y el periodo intermedio entre esos dos, en el que se identifican los principales mecanismos de transición que apoyan la conformación de la nueva identidad.

This article describes the results of qualitative research regarding teacher training of 12 professionals hired by the hour to teach courses at the Colegio de Bachilleres (equivalent to high school) in Mexico City. Based on qualitative interviews, the three professional transitions that these 12 professors have gone through are analyzed, in accordance with the proposal which was the basis for researchers at the University of Sherbrooke, experts on the subject: from an intuitive pedagogy to a systematic one; from knowledge about a discipline to knowledge with regard to how to teach it, and from an identity as a professional of different disciplines to an identity as a teacher. The development of the teacher identity is analyzed beginning with the recovery of the interviewees' career path at the Colegio de Bachilleres at three key moments: their entry as novice professors, the interview as more experienced teachers, and the intermediate period between these two moments, in which the main mechanisms that support the configuration of the new identity are determined.

\section{Palabras clave:}

identidad profesional, identidad docente, rasgos identitarios, transición, docentes, Colegio de Bachilleres

\section{Keywords:}

professional identity, teacher identity, identity traits, transition, teachers, Colegio de Bachilleres

Recibido: 22 de febrero de 2018.| Aceptado para su publicación: 24 de septiembre de 2018. DOI: 10.31391/S2007-7033(2018)0051-008

\footnotetext{
* Maestra en Ciencias con especialidad en investigación educativa. Profesora de educación media superior. Sus líneas de investigación versan sobre educación media superior y docentes. Correo electrónico: maguimmm29@ gmail.com/ https://www.facebook.com/margarita.martinezmartinez

** Doctora en Ciencias con especialidad en investigación educativa. Profesora-investigadora del Departamento de Investigaciones Educativas del Cinvestav, IPN. Sus líneas de investigación versan sobre políticas, instituciones y actores en las relaciones entre educación y trabajo. Correo electrónico: mdeibarrola@gmail.com
} 


\section{INTRODUCCIÓN}

$\mathrm{E}$

n México no existe una carrera profesional que forme a los profesores de educación media superior ni a los de educación superior (excepto la maestría en Docencia para la Educación Media Superior que se imparte en la UNAM desde 2003 y una licenciatura ofrecida por un par de universidades privadas), a diferencia de la formación especializada de nivel universitario que se exige a los profesores de preescolar, primaria y secundaria, incluso. Tradicionalmente, las posiciones docentes de las universidades y de las instituciones de los diversos subsistemas de educación media superior en México han sido ocupadas, en tiempo parcial, por profesionistas de distintas áreas disciplinares; tal es el caso del Colegio de Bachilleres (COBACH).

\section{EL CONTEXTO INSTITUCIONAL}

En 1973 se creó el COBACH como parte de la reforma a la educación media superior, que incluyó el significativo incremento de los bachilleratos tecnológicos bivalentes (agropecuarios, industriales y de servicios, de ciencia y tecnología del mar) y de los centros de educación técnica industrial y agropecuaria, así como la creación del Colegio de Ciencias y Humanidades y del Colegio Nacional de Educación Profesional Técnica.

El COBACH fue creado como organismo público descentralizado con el "objeto de impartir e impulsar la educación correspondiente al ciclo superior del nivel medio". En los hechos, su finalidad específica fue ampliar en todo el país el acceso al bachillerato general, sin dependencia de ninguna universidad para su gestión. Una vez creada, la institución vivió un periodo importante de expansión a nivel nacional, al construirse planteles del Colegio de Bachilleres en 28 estados de la república.

Respecto a la Ciudad de México y el área metropolitana, en 1973, el COBACH logró la construcción de cinco, del plantel 1 al 5; en 1978, del 6 al 16; en 1979, el 17, 18 y 19; y en 1985, el número 20. Estos son los planteles que se reportan a la fecha. La institución vivió un periodo de estabilidad y sin modificaciones en su normativa. No fue sino hasta la década de los noventa cuando comenzaron los grandes cambios estructurales: el proceso de descentralización regulado por la Ley de Educación (1993), la modificación al decreto de creación (2005), la Reforma Integral para la Educación Media Superior (2008) y la incorporación de la Ley General del Servicio Profesional Docente (2013).

\section{LA FIGURA DEL PROFESOR DEL COBACH DESDE LA CREACIÓN DE LA INSTITUCIÓN Y HASTA 2016}

La creación de todas las nuevas instituciones educativas de la reforma de los años setenta aseguró el crecimiento explosivo de la matrícula e implicó la contratación masiva de nuevos profesores para brindar el servicio. Lo anterior obligó a contratar como tales a jóvenes estudiantes, egresados de distintas carreras o disciplinas, e incluso pasantes que aún no concluían sus estudios o no estaban titulados.

En el caso del Colegio de Bachilleres, sorprende la ausencia de referencia directa a los requisitos para ingresar como profesores del Colegio, tanto en el decreto de creación de 1973 como el de su modificación. Los profesionistas sin título, contratados 
como docentes emergentes, pudieron ingresar a la docencia en esta condición (sin estar titulados) hasta 2005, año en que se empezó a exigir el título profesional. Conforme ha transcurrido el tiempo, los requisitos de contratación han cambiado; las exigencias y las vías de ingreso a las distintas instituciones educativas han sido cada vez más estrictas, incluyendo concurso de ingreso, aun en fechas previas a la puesta en marcha de la Ley General de Servicio Profesional Docente.

\section{Profesores en SERVicio}

En el panorama nacional, la docencia en el nivel medio superior se ha sostenido, tradicionalmente, con una importante proporción de profesionales de disciplinas variadas, contratados en su mayoría por horas, a los que se les denomina profesores de asignatura. Existen muy pocos datos sobre los profesores del nivel; las estadísticas nacionales informan que en el bachillerato general, $66 \%$ de los maestros trabajan por horas. Por su parte, los docentes de tiempo completo representan menos de $13 \%$ en el ámbito nacional (INEE, 2015, p. 59).

En las estadísticas del COBACH de la Ciudad de México se estiman más de cinco mil profesores y profesoras en servicio que laboran en el turno matutino, vespertino o mixto y otros más en el servicio a distancia. Sin embargo, no hay datos que hagan una clara distinción entre los tipos de contratos con los que cuentan los profesores que trabajan en la institución, pues existen los contratos de tiempo completo, medio tiempo y por asignatura, y este último tipo de contrato puede amparar una amplia diversidad de horas/semana/mes de contratación. "En razón de las necesidades educativas del Colegio de Bachilleres, el personal académico podrá laborar hasta 28 horas-semana-mes en el desempeño de la función" (Colegio de Bachilleres, 1992, p. 5). La institución dispone apenas de estadísticas generales del profesorado por plantel, no se conocen rasgos sociodemográficos ni profesionales de las características de esta figura.

\section{Problematización}

El problema a analizar es la manera como los profesores de asignatura que provienen de disciplinas ajenas al área de la educación se forman en la docencia y consolidan (o no) una identidad docente derivada de un triple proceso de transición.

De los doce docentes que entrevistamos para este estudio (Martínez, 2017), ocho fueron contratados por asignatura y cuentan ya con una antigüedad significativa de entre cinco y treinta y siete años, lo que implica que, en los hechos, en el COBACH, como en todas las instituciones que contratan profesores no formados para ello, se ha aceptado el supuesto de que es posible aprender a desempeñar el trabajo docente y todas las actividades que ello encierra por medio de la capacitación en el trabajo (ambos decretos prevén programas de capacitación y superación del personal académico), del tiempo que se dedica a la práctica efectiva de la docencia y de la interacción con autoridades, docentes y alumnos, independientemente de su formación inicial orientada hacia otro tipo de ejercicio profesional. Todas estas circunstancias generan cambios y modificaciones en el actuar de los profesionistas que se contratan como profesores, que encierran e involucran los procesos de las transiciones. 
Los profesionistas que se contratan como profesores del nivel medio superior, como es el caso de los profesores de asignatura entrevistados, no se prepararon para enseñar; durante su formación profesional no tomaron asignaturas que les aportaran elementos sobre pedagogía o didáctica en ninguno de los casos, ni siquiera los historiadores, literatos o filósofos, que son carreras que pueden desempeñar la docencia como extensión de su formación profesional.

\section{MARCO TEÓRICO Y CONCEPTUAL}

Sería erróneo plantear que la investigación se derivó de manera deductiva, ortodoxa y lineal de alguna disciplina académica en particular. Por el contrario, se genera de manera inductiva motivada por una experiencia personal real de docencia en el nivel medio superior, sin contar con la formación pedagógica para ella, y se fundamenta en un trabajo de investigación cualitativa.

No obstante, existen teorías que ayudan a analizar y explicar diversos fenómenos sociales. En la actualidad algunas contribuciones de la sociología analítica promueven la construcción de marcos analíticos, conceptos y nociones que permitan identificar diferencias y semejanzas que, lejos de interpretar, guiar, orientar los resultados empíricos de la investigación conforme a una teoría general, se inserten en un debate a partir del cual se originen diversos resultados delimitados y explicaciones fructíferas. Una de esas contribuciones son las teorías de alcance intermedio.

Al no estar fundamentadas en reduccionismos, las citadas teorías no pretenden explicar todos los fenómenos de interés sociológico; por el contrario, tratan aspectos delimitados de los fenómenos sociales, y comienzan con una idea germinal sencilla y no como una respuesta inmediata a las ideas derivadas de teorías más generales:

La noción de la teoría de alcance intermedio es considerada generalmente una de las contribuciones más importantes de Robert K. Merton a la sociología. Tal como las define el propio Merton, las "teorías intermedias (se encuentran) entre esas hipótesis de trabajo menores pero necesarias que se producen abundantemente durante las rutinas diarias de la investigación, y los esfuerzos sistemáticos totalizadores por desarrollar una teoría unificada que explique todas las uniformidades observadas de la conducta, la organización y los cambios sociales" (Merton, 1964, p. 56).

A partir de la apertura conceptual aportada por Merton, las teorías intermedias que se utilizaron para el análisis son dos: la de las transiciones (Balleux et al., 2009) y la de la identidad (Dubar, 2000; Evettes, 2003). Se aprovechó también la pertinencia y validez de las "categorías intermedias" (Buenfil, 2008), que se construyen con base en la evidencia y a lo largo del proceso de investigación.

De acuerdo con Buenfil (2008):

... la categoría intermedia es el recurso analítico que se construye precisamente para tejer los lazos que permitan transitar entre las lógicas y los conceptos ubicados en el plano teórico de lo general y abstracto, al plano de lo histórico y particular. [...] esta figura de intelección se construye ad hoc, y lo que para un objeto de estudio resulta una categoría intermedia, para otro puede no serlo. [...] es una herramienta analítica que depende de su relación con el objeto en construcción y no tiene una positividad o autonomía propias.

La categoría intermedia se produce en el desmontaje del concepto en su disciplina de procedencia para ser transferido a la disciplina educativa, por ejemplo. [...] al situarse en la tensión entre las lógicas del cuerpo teórico de procedencia y las de la particularidad 
del campo de su uso, permite un distanciamiento prudente, tanto del referente empírico (implicación del investigador) como del referente teórico ("suspensión de la teoría" Remedi, dixit 2007) (p. 33).

Además, utilizamos las siguientes categorías intermedias: condiciones institucionales de trabajo (proveniente de la sociología de las profesiones: Freidson, 1994 y Brunner, 1991, citados por De Ibarrola, 2015); el trabajo docente (Rockwell, 1985) y la satisfacción laboral (Galaz, 2003; Kellerberg, 1977).

\section{TEORÍA DE LAS IDENTIDADES}

Como síntesis de las aportaciones de varios autores, Dubar (2000), Evetts (2003), Monereo (s.f.) y Martucelli, (2010), entendemos la identidad como el proceso de (re)organización dinámica de un conjunto de rasgos propios (impulsos, capacidades, creencias, historias de vida individuales) que construyen, de-construyen y reconstruyen la imagen de sí y del otro en torno a elementos estructurantes como la valoración, la representación, el reconocimiento, la proyección (en retrospectiva) y la percepción, que surge de la interacción del sujeto en colectivo y se estabiliza o modifica al interior de los sujetos, sin importar los roles que estos asuman (estudiante, trabajador, padre, profesionista, amigo, de género, etcétera).

Según Evetts (2003), la identidad profesional, dimensión que interesa específicamente,

... se asocia con el hecho de tener experiencias, formas de entender y conocimientos técnicos comunes, así como maneras coincidentes de percibir los problemas y sus posibles soluciones. La identidad común es producida y reproducida mediante la socialización ocupacional y profesional a través de trayectorias educativas compartidas y coincidentes de la formación profesional y las experiencias vocacionales, y de la pertenencia a asociaciones profesionales (locales, regionales, nacionales e internacionales) y sociedades en las que aquellos que ejercen una profesión desarrollan y mantienen una misma cultura de trabajo (p. 32).

De particular importancia para esta investigación fueron las aportaciones de Ávila (2012), según las cuales la identidad es una relación entre elementos interactivos e interactuantes en los que la identidad de sí y del otro se construyen a partir de la semejanza y la diferencia entre elementos como el sentimiento, la imagen, la representación, la valoración, la continuidad, el ideal y lo vivido: la identidad; el sentimiento de sí tiene que ver con el nivel de reconocimiento y se refleja en la forma en que uno se ve o se imagina en lo futuro (imagen de sí).

Según Ávila (2012), la forma en la que uno se describe a sí mismo (representación de sí) tiene mucho que ver con la autovaloración que el sujeto da de su propia imagen y de su ser, que se construye a través de la interacción del sujeto con la colectividad y la comunidad; la forma en que uno se concibe como semejante a otro (continuidad de sí) posibilita el ejercicio de una práctica en la que está incluido el sí interior o colectivo, que no es otra cosa que lo que se quiere ser o lo que se quiere hacer. 


\section{TEORÍA DE LAS TRANSICIONES}

Erikson (1959, citado en Balleux y Perez-Roux, 2013), "privilegia la dimensión temporal de la transición, al considerarla un paso inevitable en la construcción de la identidad y un componente indisociable del desarrollo humano" (p. 101). Para este autor, en otras disciplinas se amplía el uso del concepto a la "movilidad de todo tipo" (de un lugar a otro, de un contexto a otro, de una época a otra, de una situación a otra...).

Lanèelle (2011) suma a estas dimensiones de espacio-tiempo la adquisición y el dominio de nuevas competencias profesionales y el compromiso con un nuevo oficio; maneja, por tanto, tres dimensiones para analizar las transiciones entre dos situaciones profesionales y, además, recupera el hecho de que múltiples transiciones operan a lo largo de una vida: "En efecto, el enseñante no puede estabilizar su identidad profesional de enseñante más que en la medida en que adquiera nuevas competencias que le permitan dominar mejor su oficio[...] ese proceso reflexivo se favorece por la integración con un grupo de pares" (Amariya y Mukamuera, 2005, citados por Lanèelle, p. 95, cursivas nuestras).

Balleux, (2009) y Balleux y Perez-Roux (2013) conceptualizan la noción de transición para identificar los elementos fundamentales que permiten analizar los cambios en la identidad; la proponen como una transposición en el sentido de pasar a través de un espacio y, sobre todo, trascender; también en el sentido de idea del tiempo que transcurre o como un intermediario entre dos estados, dos situaciones y no necesariamente un cambio de espacio, como ha sido frecuente en estos estudios. Así, en el fundamento mismo del concepto se perciben las dimensiones de espacio y de tiempo, que aparecen imbricadas, periodos de pasaje entre dos estados.

Este escenario nos permite analizar el proceso que atraviesan los profesionistas que se convierten en profesores de asignatura; proponemos que este proceso se caracteriza por la experimentación de distintas "transiciones".

A partir de las aportaciones de los autores citados, identificamos tres tipos de transiciones para los profesores de asignatura del COBACH: (el pasaje) de una pedagogía intuitiva a una más sistemática; de un conocimiento para ejercer una profesión a uno disciplinario para ser enseñado en el nivel medio superior; y de una identidad profesional ajena a la docencia a una identidad docente. Cabe mencionar que en el apartado de los resultados describimos en detalle cada transición.

El indispensable análisis de la dimensión tiempo de las transiciones, unido a la estrategia metodológica de las entrevistas, condujo a precisar tres momentos fundamentales en la trayectoria de los profesores:

-El ingreso al COBACH como profesores de asignatura (a partir de la manera como lo recuerda el profesor mediante su narrativa).

-El momento más reciente que corresponde al de la entrevista, cuando los profesores nos hablan del dominio que han adquirido sobre una pedagogía más sistemática y del tipo de conocimiento que manejan con los alumnos.

-Entre estos dos momentos fue posible identificar un "periodo intermedio" y apuntar diversos elementos que encierra el aprender a ser maestro, periodo en el que intervienen las condiciones laborales, el acompañamiento institucional, múltiples mecanismos, así como la interacción con personajes clave. 


\section{DECISIONES METODOLÓGICAS}

El trabajo de campo para esta investigación se realizó en 2015:

Un enfoque cualitativo

Dada la riqueza de los enfoques teóricos encontrados para acercarse a este problema, consideramos necesario plantear un estudio cualitativo que pretendió profundizar en la realidad que viven doce profesores contratados por asignatura del COBACH, así como la manera en que recuerdan su ingreso a esta institución y recuperan su paulatina transición hacia la docencia (Miles \& Huberman, 1994; Ezzy, 2002).

\section{La entrevista}

Fue el instrumento fundamental de investigación, ya que permite entender distintas realidades, acercarnos a comprender fragmentos del mundo social y la forma de interacción de los sujetos en contextos específicos (Maccoby \& Maccoby, 1954). En esta interacción cara a cara con los entrevistados fue indispensable poner especial atención en dos dimensiones: por una parte, el ámbito profesional original de los entrevistados (su formación profesional inicial y su trayectoria laboral) y, por otra, las transiciones hacia la docencia: reflexiones de los entrevistados sobre su ingreso a la docencia, condiciones laborales, mecanismos de acompañamiento institucional, práctica docente dentro del COBACH, además de la configuración de una identidad docente.

\section{Criterios para la selección de planteles}

De los 20 planteles del Colegio de Bachilleres que existen en la Ciudad de México y área metropolitana, seleccionamos dos a conveniencia, por su ubicación y acceso: el número 15, Contreras, y el 17, Huayamilpas. Solicitamos la autorización de la directora general para invitar a los profesores a participar en este estudio y también nos comunicamos con los directores de los planteles para pedir su autorización.

\section{Criterios para la selección de doce profesores}

Entrevistamos a doce profesores de asignatura, elegidos en función de la asignatura que imparten, su antigüedad en el Colegio y su formación profesional. Estos profesores fueron seleccionados considerando que no buscamos formular una generalidad, y en el entendido de que no son una muestra representativa; lo que nos interesó fue el acercamiento que tuvimos y la oportunidad de escuchar sus voces para conocer su realidad de cerca, tal como ellos la perciben.

\section{El complemento documental}

Retomamos documentos oficiales fundamentales para la investigación: el decreto de creación de la institución de 1973; el decreto de modificación de 2005; el acuerdo 447, de octubre de 2008, en el cual se muestra el nuevo perfil docente para todos los subsistemas del nivel medio superior; el documento de la Reforma Integral para la Educación Media Superior de 2008; el mapa curricular vigente del COBACH y la tabla de perfiles profesionales de sus profesores, entre otros. Estas fuentes nos permitieron efectuar una triangulación respecto a la información empírica que proporcionaron los profesores entrevistados. 


\section{RESULTADO DE LA INVESTIGACIÓN}

La narrativa de los profesores entrevistados ofrece la evidencia de las transiciones hacia la docencia que vivieron estos profesionistas contratados como profesores de asignatura, la cual se organizó en cuatro grandes resultados: el ingreso al COBACH y las condiciones laborales de los profesores; las expresiones de las transiciones; los mecanismos que intervienen en estas transiciones; y la consolidación de una identidad docente.

\section{El ingreso al COBACH y las condiciones laborales de los profesores (ver apéndice)}

$\mathrm{Al}$ hablar de las transiciones, destaca el primer punto, el ingreso: "Cuando se comienza a desarrollar una actividad laboral, más aún cuando se realiza por primera vez, se vive un periodo particularmente difícil. En esta etapa se mezclan las expectativas, las ilusiones y las esperanzas con los desconciertos, el desánimo, el desaliento" (Beca y Boerr, 2009, p. 109); podríamos agregar que estas emociones se exacerban ante la falta de una formación profesional que dé confianza para el desempeño docente.

En este momento del ingreso fue posible clasificar a los profesores en dos rubros: los profesores que nunca habían estado frente a grupo y los que tenían experiencia previa en la docencia.

\section{-Profesores sin ninguna experiencia docente}

Para cinco profesores entrevistados, el ingreso a la docencia en el COBACH representó su primera experiencia frente a grupo y no para todos fue placentera. En este sentido, manifestaron distintas percepciones de cómo vivieron su ingreso al COBACH; una de las profesoras expresó sus sentimientos respecto a sus carencias en el ámbito educativo y la indiferencia de la institución al iniciarse en esta labor, no solo por no saber nada o muy poco sobre el ámbito pedagógico y didáctico, sino porque, aunque está formada en un área profesional, desconocía ciertos temas propios de la disciplina y, lo más importante, no sabía cómo desempeñar el papel de profesora:

O sea, yo no tenía ninguna experiencia en docencia, tú nada más traes los conocimientos de la universidad, donde dices a lo mejor dar una clase es como dar una exposición que tú haces frente a tus compañeros ¿No? Entonces este..., pues así. Me dice: y mañana ya tiene su primera clase con su grupo. Entonces, yo me quede así ¿y ahora qué? Me da un programa de estudios, me da un horario y me dice: mañana se presenta... (R10, 2015).

-Profesores con experiencia docente previa

Siete profesores entrevistados reportaron experiencia previa en la docencia en distintos niveles educativos: en preescolar, secundaria, preparatoria, nivel superior y en educación para adultos, aunque ninguno tuvo una formación profesional para ello. No obstante, esta experiencia les permitió que su incorporación al COBACH fuera más fácil, porque ya habían aprendido varios elementos que encierra el oficio de ser profesor y vivieron el proceso de la iniciación en la docencia en otras instituciones.

Sin embargo, también vivieron un periodo de adaptación dado que la lógica institucional cambia de una escuela a otra (normativa, funcionamiento, tipo de contrato, horarios, asignaturas, entre otros elementos, cambian), y también el proceso de aprender a ser profesores del COBACH: 
Bueno, creo yo que me adapte rápidamente, o sea cuando yo empecé a dar clases en el Colegio de Bachilleres, pues para mí fue una impresión un poco triste porque yo venía de dar clases en una escuela particular y el nivel socioeconómico era muy diferente, pero pues me di cuenta que aquí podía ayudar mucho, digo relativamente a lo mejor es un granito de arena para el colegio $(\mathrm{R} 8,2015)$.

-Condiciones de trabajo

Aunado al complicado inicio como docentes, estos profesores han tenido que enfrentarse a difíciles condiciones de trabajo: los doce profesores entrevistados están contratados por asignatura, ese fue el criterio básico de selección para la muestra, pero el contrato es distinto para cada uno de ellos, pues las contrataciones varían entre tres, cuatro o cinco horas a la semana y hasta veintiocho, que es el número máximo por las que pueden ser contratados. Todos ellos aceptan, de alguna forma, las condiciones laborales que representa un contrato de esa naturaleza, porque, finalmente, salvo uno, cuentan con una amplia antigüedad en ese cargo, pero expresan ciertas reservas o desacuerdos.

Para los profesores de asignatura contratados por tres, cuatro, cinco o siete horas semanales (cuatro profesores entrevistados), la relación con el COBACH no constituye su primera fuente de ingreso ni su trabajo principal. Sin duda, no es necesario hacer hincapié en que estas condiciones de trabajo no permiten al profesor de asignatura considerar a la institución como su sustento de vida o como su prioridad en el comparativo con su otro empleo.

Por otro lado, los profesores que están contratados por veintiocho horas perciben mayores ingresos, aunque varios de ellos también tienen otro empleo. No obstante, las variables en sus contratos con el COBACH pueden ser conjugadas de diferentes maneras y estas pueden ser muy adecuadas para las necesidades del profesor o muy complicadas.

El significado del número de horas de contrato no se agota en una cifra; la valoración que hace el profesor pasa por los siguientes hechos: cuántas asignaturas diferentes debe impartir en un mismo semestre; de qué semestre son: $1^{\circ}, 3^{\circ}, 5^{\circ}$, o bien, $2^{\circ}, 4^{\circ}$ o $6^{\circ}$, según sea el caso, la cantidad de grupos que debe atender; la concentración o dispersión de sus horas de trabajo; secuencia, o no, en los días de la semana que debe acudir al plantel; y el trabajo diferenciado que implican esos escenarios marcados en el contrato:

... por eso te digo, son seis materias diferentes y es lo que les comentaba: ¿cómo le voy a hacer para preparar seis clases diferentes? ¿A qué hora? Imparto Física I, II, III, Física I del plan anterior y TIC II y IV (R2, 2015).

... es más, hay compañeras que tienen dos grupos de Ecología, dos de Biología, y a lo mejor tres grupos de Química y así es más complicado porque tienen que tener bien presente lo de Química, Biología y Ecología y así es más complicado (C12, 2015).

\section{La expresión de dos de las tres transiciones analizadas}

En este apartado identificamos, en palabras de los entrevistados, la expresión de dos de las tres transiciones que hemos sistematizado a partir de las aportaciones de los autores de referencia: de una concepción pedagógica intuitiva a una concepción derivada de una 
pedagogía más sistemática, y de un saber para ejercer una profesión a un saber a ser enseñado como parte de una formación general de bachillerato.

Estas dos primeras transiciones se analizan de manera conjunta, pues son difíciles de separar y delimitar, dado que la pedagogía y la transmisión del conocimiento se relacionan estrechamente, aunque el profesor no las domine por completo.

Al momento de la entrevista, cada profesor narró su recuerdo de la experiencia de su inicio en la práctica docente, sus características, sus escasos referentes didácticos y pedagógicos y sus limitantes; sin embargo, con esas particularidades y carencias el profesor debía trabajar en el aula (trabajo para el cual fue contratado), tratar de cubrir el programa, atender a los alumnos, enseñar y afrontar los obstáculos que se le presentaran en el contexto escolar e ir resolviéndolos en la medida de lo posible (aunque ningún profesor entrevistado habló de tiempo límite impuesto por el COBACH para mejorar en su desempeño docente):

... es más en principio me costó mucho trabajo porque las clases eran de dos horas. Entonces imagínate principiante, sin saber cómo, pues a mí me sobraba mucho tiempo y pues luego los prefectos estaban aquí, te están checando que empieces a tu hora y terminaras a tu hora, pues yo tenía infinidad de reportes ¿no? porque a mí se me hacía fácil, digo: pues si ya había terminado, pues los dejo salir ¿no? Y pues a veces era la media hora antes de las dos horas de sesión, si no eran los veinte minutos, pero pues porque yo no sabía planear una clase ¿no? Lo que se necesita hacer para distribuir los tiempos y te alcanzará ¿no? (R10, 2015).

En el ingreso, podemos caracterizar al profesor de asignatura como aquel sujeto que tiene una formación alejada de la educación; no tiene experiencia en la docencia o muy poca; sin conocimientos de cómo planear ni impartir una clase; utiliza sus referentes inmediatos (intuitivos, empíricos) que hacen alusión a la experiencia que tuvo como estudiante; recuerda las prácticas que tenían sus profesores; no cuenta con una amplia gama de estrategias pedagógicas ni didácticas (se apoya de manera constante en el dictado); no ha recibido capacitación alguna para realizar su trabajo docente, ya sea institucional o voluntaria ni tampoco hace un uso efectivo del tiempo, y su conocimiento profesional es la herramienta más importante que posee, aunque no lo tiene adecuado a un plano para ser enseñado a los alumnos de bachillerato, sobre todo por el lenguaje que utiliza (tecnicismos propios de su formación profesional); no se pregunta todavía por los diferentes tipos de alumnos que debe atender en el Colegio ni por las posibles diferencias que presentan los alumnos de los distintos turnos y el tamaño de los grupos.

En los hechos, los profesores imparten clases inicialmente a partir de su intuición de lo que es enseñar y se apoyan en el conocimiento profesional que poseen de su formación inicial, sobre todo en los primeros momentos del ejercicio docente:

Yo traté los primeros inicios de darlo casi como a nivel universitario o sea como un nivel más elevado, muy elevado, entonces al inicio sí tuve [...], la primera evaluación tuve muchos reprobados, porque no [...], les faltaban muchas bases a ellos todavía, al nivel de $[\ldots]$, que yo lo trataba de manejar el tema... $(\mathrm{F} 3,2015)$

Al rememorar su experiencia como docentes, los profesores resaltan seis elementos de los que han carecido, que entorpecen y dificultan su práctica en el contexto escolar: el proceso de planeación de la clase cotidiana, del semestre y la falta de experiencia para elaborarlo; el uso del tiempo; la carencia de estrategias pedagógicas y 
didácticas; el uso adecuado del lenguaje (lenguaje docente, lenguaje profesionista); la identificación de las necesidades de los alumnos; y el manejo de grupo.

Estos elementos van entrelazados: el proceso de planeación y el uso eficiente del tiempo van de la mano, al igual que el empleo de estrategias didácticas y pedagógicas para abordar un tema y elaborar una planeación. Los elementos como el uso del lenguaje, el manejo de grupo y la identificación de las necesidades del alumno son más operativas, porque se hacen presentes durante el desarrollo de la clase; estas carencias dificultan el trabajo desde la planeación hasta la ejecución.

Durante la entrevista, los profesores reportan los nuevos aprendizajes adquiridos. Para este momento ya aprendieron a realizar planeaciones, seleccionar las actividades que van a llevar a cabo en el aula, ensamblarlas entre sí y con los tiempos que requiere; esto último es fundamental: aprenden a usar de manera eficiente el tiempo y a dosificarlo, ya no les sobra ni les falta; dejan el dictado constante y han adquirido una gama de estrategias didácticas y diversas técnicas; hacen su clase más dinámica e implementan nuevas formas de trabajo; aprenden a utilizar un lenguaje que los adolescentes entienden; encuentran maneras más claras y simples de explicar los temas; aterrizan contenidos en el contexto de los alumnos; asimilan que tienen distintos tipos de alumnos y son capaces de diferenciar las características y necesidades que tienen; y aprenden a trabajar en grupos grandes y pequeños, en turno matutino y vespertino.

Los anteriores son los seis elementos identificados por los profesores entrevistados como los problemas que más los impactaron y que no pudieron resolver eficientemente al inicio de su práctica docente, aunque no son los únicos, porque también están los problemas que intervienen en la planeación, su puesta en práctica o su consistencia, así como los procesos de evaluación del aprendizaje de los alumnos. De la misma manera, afectan a los profesores los eventos no previstos, que surgen en las interacciones con los alumnos, padres de familia y jefes directos, que, en un primer momento, no saben cómo manejar.

Después de haber advertido estas carencias en el desempeño de los profesores, identificamos procesos y mecanismos que ayudan a mejorar su desempeño en el aula y que son fundamentales en los procesos de las transiciones.

\section{Procesos y mecanismos que influyen en las transiciones}

En este apartado mostramos los procesos y mecanismos que propician e influyen en los cambios (las transiciones) que han tenido los profesores en el desempeño de su trabajo como docentes, entre ellos destacan la capacitación en diversas modalidades (institucional, voluntaria, en otros espacios), la práctica cotidiana y la interacción con diversos personajes clave.

-La capacitación

La capacitación en sus diversas modalidades es un mecanismo fundamental; la capacitación institucional (presencial o en línea), voluntaria y la que recibieron en otros espacios laborales puede ser vista como piedra angular en el proceso de aprender a ser profesor. "Muchas veces el discurso sobre la formación docente (sobre todo post 
inicial) la plantea como una varita mágica que logrará resolver las cuestiones más simples y también las más difíciles a las que se encuentran enfrentadas la sociedad y la escuela" (Birgin y Dussel, 1999, citado en Birgin, 2012, p. 14) En los procesos de capacitación, el profesor encuentra herramientas para desempeñar su labor en el aula, pero hay otros elementos que complementan la formación del profesor, pues la capacitación por sí misma no puede solucionar todos los problemas que se viven en la escuela.

\section{- La capacitación institucional}

El COBACH ha instituido los cursos intersemestrales que ofrece a los profesores con un límite de hasta tres por profesor; tienen distintas duraciones y pueden ser presenciales o en línea. La capacitación prepara/forma/profesionaliza al profesor para que desarrolle habilidades y conocimientos que le serán útiles en su espacio de trabajo. La capacitación que brinda la institución es fundamental para el desarrollo y la consolidación de profesores más expertos. Sin embargo, en los planteles, la capacitación no funciona con tal precisión ni funciona como la cura de todos los problemas de los profesores y de la institución; no siempre hay congruencia entre las asignaturas que imparten los profesores y la capacidad de elegir los cursos adecuados:

Yo fui formada como docente en el Colegio, en el intersemestral enero-febrero, juniojulio se nos paga para que estemos en continua actualización, el Colegio tenía hace muchos años tres áreas: la psicopedagógica, la pedagógica y el área disciplinar. Entonces tú tenías un abanico de posibilidades para formarte (X9, 2015).

El curso fue lo que alcanzaste a tomar, ahí te inscribiste porque se saturan muy rápido, nosotras como nuevas el día de las inscripciones nuestras matrículas no entraban en la computadora, no nos pudimos inscribir. Entonces, la jefa de materia mandó un correo a la Dirección General y ellos nos inscribieron al curso que quisieron. Tuve que tomar el curso de química siendo que aquí no doy química, yo doy ecología, el semestre pasado di biología (C12, 2015).

- Capacitación voluntaria

Como parte del proceso de consolidación como profesor experto, cinco profesores entrevistados tuvieron la iniciativa de capacitarse de manera voluntaria, porque la consideraron necesaria para mejorar su práctica docente; a pesar de los diversos obstáculos que puedan enfrentar, buscaron los recursos de tiempo y dinero para hacer posible su crecimiento profesional.

-Capacitación en otros espacios

También está la capacitación que han tenido en otros espacios laborales y que trasciende el espacio del COBACH. Hay que recordar que siete profesores entrevistados tenían experiencia previa en la docencia antes de ingresar al COBACH, situación que les ha permitido tener capacitación en otros espacios laborales; aunado a lo anterior, siete de los profesores entrevistados desempeñan un segundo empleo ligado al ámbito educativo, lo que les abre otros espacios para ser capacitados.

- La práctica cotidiana en el aula

Además de las múltiples herramientas de las que pueden echar mano los docentes, es importante darle el valor que merece a la práctica constante. La escuela es, de hecho, el lugar privilegiado de la formación permanente del maestro (Fierro, 1999, 
p. 22.), ya que en él el profesor llevará a cabo ciertas prácticas continuas (trabajo en el aula que se refiere a enseñar usando técnicas expositivas, diseñar actividades, interactuar con los alumnos, calificar tareas, realizar exámenes, etcétera), no solo en el sentido de repetirlas, sino de perfeccionarlas o mejorarlas y utilizarlas de una manera más eficiente: “... la verdad es que dicen que la práctica hace al maestro (J5, 2015). Sales de una carrera, de una licenciatura, pero, pues no sales siendo maestro de ahí. Entonces lo vas aprendiendo conforme a la práctica, para poder ser maestro" (R10, 2015).

\section{-Las prácticas innovadoras en el aula}

Rockwell (1988) denomina estas prácticas como lo "anormal" e incluye en esa denominación desde prácticas excepcionales o aun reprimidas, como las de organizar cierto tipo de juegos hasta otras que corresponden poco al "deber ser", como preparar clases dentro del salón o leer revistas mientras los alumnos hacen su trabajo (p. 67). Por fortuna, las prácticas innovadoras que narraron los profesores entrevistados están relacionadas con el lado positivo de esas prácticas excepcionales.

En el trayecto más o menos largo como docentes, los profesores entrevistados aprendieron a impartir clases aplicando y reflexionando sobre los modelos y programas formales, pero también hay algunos que se atreven a innovar, a llevar a cabo prácticas poco comunes que surgen precisamente porque no se formaron como maestros; por lo tanto, no están casados por completo con los modelos y las formas tradicionales de impartir clase. Se siguen los guiones (planeación) en menor o mayor medida y se respetan ciertos parámetros, pero las actividades que algunos profesores llevan a cabo son innovadoras, sorprenden a los alumnos y los conectan con el mundo en que viven, aprovechando su formación profesional.

Varios profesores, desde su formación profesional, han sido capaces de crear y recrear nuevos escenarios para que los alumnos puedan tener otras formas de aprender, como a elaborar helados en el laboratorio de química, ocupar nuevos espacios o materiales, o bien, enseñarles a crear o a imaginar nuevos contextos. Según sus relatos, existe una desvinculación entre la escuela y la vida real y, a veces, los jóvenes no palpan la realidad en la que viven:

... digo que el trabajo en el aula es importante, pero muchos de ellos a la mejor nunca han sembrado una planta, les hablas de una planta pero digo bueno nunca la han sembrado, nunca la han cuidado, nunca la han visto germinar; entonces como que tienes que enfrentarlos más a lo que es real, a lo que [...] o sea de veras a [...] entonces ahorita vamos a organizar, vamos a agarrar el jardín de cactáceas que está aquí atrás, los chicos van a tener que venir, van a tener que estar, van a tener que plantar, van a tener que regar ¿Por qué? Porque a veces son cosas que no hacen, entonces también es importante la parte del conocimiento teórico y muy científico, pero también les hace falta a veces vivir un poquito más la realidad $(\mathrm{C} 12,2015)$.

-La interacción con sus pares

La interacción entre pares es otro mecanismo que interviene en la formación del profesor más experto. Cuando se trata de hablar de la manera en la que el profesor del COBACH se forma, las interacciones entre pares (profesores) ocupan un lugar privilegiado, dado que en esos momentos de interacción (formal o informal) comparten experiencias (anécdotas de cómo enfrentar alguna situación, consejos, técnicas de 
trabajo), formas de trabajo, ejercicios, material didáctico y esta situación permite retomar elementos que sirvan para mejorar su práctica en el aula:

Y lo más interesante de ahí es que convives con otros maestros, que tienen más años que tú en la docencia y además en ese momento se da la situación de que ellos te explican cómo abordar ciertas situaciones. Entonces te enriquece eso, aprendes de los maestros que ya tienen experiencia, de repente ves sus situaciones y ves los problemas que tu tuviste, a veces similares, la forma en como lo resolvieron ellos, como lo resolviste tú, dices: ¡rayos! Es eso lo que me falló y entonces la ventaja de convivir es eso (J7, 2015).

No obstante, es ahí donde el número de grupos que debe atender un profesor, sus horarios y calendarios pueden entorpecer las posibilidades de interacción con sus colegas.

Ningún elemento de los que hemos mencionado por sí mismo puede remediar o solventar todas las carencias de un profesor; cada elemento ocupa un lugar importante en la consolidación del profesor más experto, en el que se han convertido los entrevistados; sin embargo, reconocemos que cada profesor aprende de manera distinta, usa de modo diferente sus recursos y los tiempos son disimiles para cada uno de ellos.

\section{La tercera transición: de una identidad profesional (ligada a una disciplina ajena a la educación) a una identidad profesional docente}

Después de hablar de los mecanismos que intervienen en las transiciones, llegamos a la tercera transición: de una identidad profesional a una identidad docente, lo que nos permite describir rasgos fundamentales que intervienen en el cambio o la consolidación de una identidad profesional, considerando los múltiples factores que se ponen en juego para lograr una construcción identitaria. En este apartado abordamos la manera en que el profesionista-profesor se autopercibe, cómo se reconoce y se autonombra, como lo abordamos en la presentación teórica, de acuerdo con Ávila (2012).

Para consolidar una identidad profesional, se necesita tiempo (incuantificable); por lo tanto, el profesionista que se contrata como profesor, cuando ingresa al COBACH, llega con una identidad profesional (inicial), marcada por el tiempo que duró su formación universitaria, los años que se dedicó a ejercer una profesión; sin embargo, no es la misma situación para todos, pues otros llegan con una identidad docente incipiente; por ejemplo, como ya lo mencionamos, siete profesores entrevistados ya tenían distinta experiencia en la docencia, situación que pudo contribuir al inicio de una posible transición identitaria.

Es notorio que los profesores con una trayectoria más larga dentro de la institución muestran un apego más fuerte a la docencia y, en cierto modo, un mayor sentido de pertenencia a la institución en la que laboran, así como mejores condiciones de trabajo. Aunque no percibimos un arraigo como este entre los profesores de recién ingreso (que durante la entrevista tenían meses o semanas dentro de la institución), estos sí pensaban continuar en la docencia y obtener más horas frente a grupo en los siguientes semestres.

Con el paso del tiempo (trayectorias dentro del COBACH) y de acuerdo con los factores y mecanismos descritos, tenemos tres posibilidades respecto a la construcción identitaria de los profesores de asignatura del COBACH: 
- El arraigo a su profesión inicial

En el bachillerato, la especialidad por materias y áreas del conocimiento se sobrepone a las obligaciones que como educador se supone que contrae todo profesor. Es fundamental hacer hincapié en que los procesos de contratación en el COBACH reafirman la importancia de la relación que existe entre la formación inicial de los profesionistas contratados y la asignatura que imparten, situación que privilegia la convivencia entre profesores que provienen de su mismo gremio profesional.

Para dos profesores, el ingreso a la docencia representó un choque entre el papel que desempeñaban en sus antiguos empleos: en la industria o en otras instituciones públicas o privadas, y el nuevo papel que han tenido que asumir como docentes:

No soy maestro, no tengo esa profesión de formación inicial digamos como los normalistas, ellos sí son ahora sí que docentes, yo soy un profesional, diferente a ser maestro $(J 7,2015)$.

No. Definitivamente yo me siento química y de hecho mi experiencia se la platico a mis alumnos y siempre hacemos analogías de porque es importante $\mathrm{X}$ o Y tema y porque lo van a utilizar y no importa si no son químicos (S4, 2015).

Ambos siguen considerando en su narrativa una fuerte pertenencia a su gremio profesional; podría suponerse que esta situación se da por el tipo de convivencia que se origina dentro de la institución y el contenido de las asignaturas que imparten, que está muy relacionado con su formación inicial. En la realidad existe una contradicción en su discurso, pues se dedican a la docencia, ya que su otro empleo también está centrado en esta, además de que no desempeñan actualmente ningún trabajo especializado de su profesión inicial.

\section{La transición hacia una identidad profesional docente}

Tres profesoras se autonombran de asignatura; no se reconocen como licenciadas. Cabe mencionar que sus trayectorias van desde los doce hasta los treinta y siete años dentro del COBACH. Si consideramos que la pertenencia a un grupo social implica no solo formar parte de él, sino asumirse como parte de él y adjudicarse las consecuencias desfavorables, o bien, obtener los beneficios que este le ofrece (Bornewasser \& Bober, 1987), esta identidad envuelve una autoadscripción. La antigüedad dentro de la institución, el trabajo que desempeñan, los mecanismos que intervienen en su práctica cotidiana, los procesos de capacitación, pero en particular "la satisfacción laboral que les genera su trabajo", el reconocimiento que les da la institución y la importancia que otorgan al reconocimiento por parte de los alumnos a su labor docente son elementos fundamentales para consolidar su identidad docente:

Sí, o sea yo desde ahorita la verdad digo lo mío, es la docencia. Sí. Sigo en contacto con la veterinaria, bueno tan en contacto que sí, a mis alumnos les da mucha risa porque dicen ¿tiene mascotas? Sí, les digo tengo cinco perros, tres gatos, un hurón, un marido y una hija y todos "ay maestra", les digo sí tengo muchos perros y me gustan; pero no, prácticamente lo mío ya es la docencia $(\mathrm{C} 12,2015)$. 
Soy maestra, definitivamente historiadora ya no me puedo sentir porque ya no hago investigación (B1, 2015).

No me considero licenciada, maestra sí (R10, 2015).

De igual modo, podemos considerar que algunos profesionistas habían iniciado previamente la transición hacia una identidad docente porque más de la mitad de los que entrevistamos ya contaban con experiencia previa en la docencia; uno de ellos, incluso, el profesor JA5-, lo respalda una trayectoria muy amplia en docencia en el nivel medio superior y superior, antes de ingresar al COBACH.

\section{Consolidación de una doble identidad}

El devenir del tiempo y la permanencia de los profesores dentro de la institución van marcando y modificando su desempeño; dejan de hacer un trabajo especializado respecto a su profesión y se van desprendiendo de las actividades para las cuales fueron formados inicialmente, aunque no del todo; por ejemplo, el contador deja de hacer contabilidades; su trabajo ahora consiste en enseñar a los alumnos cómo se realizan esos procesos; el ingeniero químico ya no trabaja en la fabricación de productos, ya que ahora su trabajo consiste en enseñar procesos químicos, se desempeñan como docentes.

Al tiempo que la misma lógica del trabajo docente los va permeando, la relación que existe entre las asignaturas que imparten y su formación inicial sigue siendo muy fuerte y la convivencia con profesionistas de su mismo gremio está presente. La forma institucional del trabajo por academias ocasiona que profesionistas de una misma disciplina se congreguen en el interior de la institución; esta situación les genera un sentido de pertenencia a su área disciplinar, aunque en otras condiciones; podría decirse que se fortalece una identidad profesional por áreas disciplinares a la par que se asume una identidad como profesores de asignatura del Colegio de Bachilleres y todo lo que implica.

De los profesores entrevistados, siete se reconocen con dos identidades profesionales: por un lado, conservan su identidad profesional, sienten un gran arraigo a su gremio profesional y, por otro, asumen su identidad docente, no solo por el rol que desempeñan, sino porque sienten pertenencia:

Yo me considero ambos, tanto maestro [...] como ingeniero ¿no? Porque he tenido la oportunidad de estar en las dos partes [...] y me siento como ingeniero y como docente $(\mathrm{R} 2,2015)$.

Me siento ambos, me siento un ingeniero con actividades docentes $(\mathrm{R} 8,2015)$.

Las dos cosas se conjuntan porque como docente tengo que administrar también [...], entonces es parte de lo que aprendí en la carrera lo estoy aplicando para mi planeación, he hecho planeaciones con lo que se inicia la administración y aquí vemos planeación didáctica, también es lo que iniciamos, entonces las dos se van complementando de alguna forma y luego cuando hay reuniones así heterogéneas que van de todos los profesores. Entre los administradores si ves, si no saben planear, como siendo administradores no saben planear, no saben organización porque es parte de lo que tenemos como formación y lo aterrizamos a la parte docente, entonces no puedo decir que tenga más, quizá en este momento tenga más la docencia porque 
incluso ya no hablo con términos bursátiles, sino ahora hablo de listas de evaluación, rubricas, enseñanza, capacitación y todo eso... (F3, 2015).

Como lo señala Martucelli (2010), la identidad se puede identificar por el rol que desempeñan; es entonces cuando podemos nombrar y ubicar a estos profesionistas como profesores de asignatura del Colegio de Bachilleres, por el rol que desempeñan, acompañados del tipo de contrato que poseen (por asignatura) y por su trabajo dentro del aula. Sin embargo, la mayoría de los entrevistados asumen una doble identidad profesional. A diferencia de lo que plantea Balleux et al. (2009), esta última transición no se cumple en todos los casos: siete profesores entrevistados asumen ambas identidades, viven una dualidad, porque nunca dejan de lado la profesión inicial completamente; gran parte del conocimiento que tendrán que enseñar lo aprendieron en su formación profesional inicial; por otra parte, el desempeño y su autoadscripción como docente nos muestran la construcción de una doble identidad y, marginalmente, la resistencia a identificarse como profesor.

\section{CONCLUSIONES}

Los profesores de asignatura presentan una enorme diversidad ante la docencia en una institución de nivel medio superior. Además de la diversidad por sexo, edad y profesión de origen, tienen contratos de temporalidad muy diferente: tres, cinco, diez, doce, quince o hasta veintiocho horas; en este sentido, el número de horas contratadas por semestre debe conjugarse con otros datos de la contratación: el número de grupos, entre uno y nueve; el número de asignaturas diferentes, entre una y seis; el tamaño de los grupos; los semestres en los que se imparte; y los turnos que laboran. Esta situación genera sentido de pertenencia e influye en los procesos de la transición.

Por otra parte, las transiciones hacia la docencia analizadas son complejas y nuestra intención no es que se entiendan como dos puntos precisos en el tiempo; no se pueden explicar como procesos perfectos y lineales. Un dato que no podemos olvidar es que los profesores tienen antecedentes profesionales distintos y antigüedades muy diferentes en el ejercicio docente: de mes y medio a treinta y siete años.

Cabe destacar que es un mismo sujeto el que vive las distintas transiciones, y cada mecanismo y cada proceso lo percibe de distinta forma; los tiempos para que sucedan los cambios son imprecisos y distintos para cada uno. Por lo mismo, más que analizar trayectorias en su individualidad, nuestro objetivo era mostrar los elementos que los profesores coinciden en identificar como los más importantes a superar en su desempeño como docentes: la planeación de la enseñanza por clase y por semestre; el uso eficiente del tiempo y de las estrategias pedagógicas y didácticas; el uso del lenguaje; el manejo del grupo; y la posibilidad de identificar diferencias entre alumnos al interior de un grupo y entre los grupos que atienden.

Las transiciones son producto de diversos procesos complejos, en los que intervienen mecanismos institucionales y personales sin los cuales no podrían llevarse a cabo. Destaca la importancia de la capacitación institucional y la interacción con los otros profesores, pero, a la vez, son muchos los obstáculos que imponen las formas y condiciones de contratación. Varios profesores tienen la iniciativa personal de 
mejorar su práctica profesional y destinan recursos propios y tiempo para ello. $\mathrm{Su}$ práctica cotidiana no solo les fortifica su identidad docente; se nutre también de su formación disciplinaria inicial y, lo más importante, es fuente de satisfacción en el trabajo por el reconocimiento que reciben de sus alumnos.

Por último, las transiciones que fueron analizadas hacia una nueva identidad no se resuelven en todos los casos por el abandono de una identidad a favor de otra, sino que ambas identidades se alimentan mutuamente.

La investigación realizada aporta contenidos puntuales a lo que se denomina pedagogía intuitiva, que se desprende de las vivencias no formales de contacto con la enseñanza que vivieron los sujetos, antes de su ingreso al COBACH, e identifica por lo menos seis rubros propios de la pedagogía sistemática, la cual aprendieron los profesores por muy diversas vías, en particular por su experiencia. A lo largo de su trayectoria, descubren que no es lo mismo saber una disciplina que saber enseñarla, asunto que ha llevado a desarrollos tan difíciles en la formación de profesores como las didácticas específicas.

La metodología cualitativa utilizada no nos permite generalizar los resultados, pero la identificación de los problemas que experimentan los profesores de asignatura que provienen de disciplinas ajenas a la enseñanza aporta elementos muy pertinentes para el contenido de los cursos de capacitación docente y para la programación de los tiempos y espacios de trabajo colegiado que requiere la mejor atención a los alumnos del nivel.

\section{REFERENCIAS BIBLIOGRÁFICAS}

Ávila, M. (2012). Intervención pedagógica, campo profesional e identidad pedagógica. Recuperado de http://www.acatlan.unam.mx/multidisciplina/ file.../126/multi-2012-01-05.pdf

Balleux, A. et al. (2009). La transition entre le métier et l'enseignement des nouveux enseignents de formation profesionnelle: un passage a comprendre et a accompagner. Proyecto de investigación.

Balleux, A. y Perez-Roux. T. (2013). Alrededor de las palabras de la formación. "Transiciones profesionales". Universidad Paul-Valéry-Montpellier 3, LIRDE.

Beca, C. y Boerr, Ingrid (2009). El proceso de inserción a la docencia. El difícil comienzo. En D. Vaillant (coord.). Aprendizaje y desarrollo profesional docente. España: Fundación Santillana.

Birgin, A. (comp.) (2012). Más allá de la capacitación. Debates acerca de la formación de los docentes en ejercicio. Buenos Aires, Argentina: Paidós.

Bornewasswer, M. \& Bober, J. (1987). Individual, social group and intergoup behaviour. Some conceptual remarks on the Social Identity theory. European Journal of Social Psychology, núm. 17, pp. 267-276.

Buenfil, R. (2008). La categoría intermedia. En O. Cruz y Laura Echevarría (coords.). Investigación social. Herramientas y análisis político del discurso. México: Casa Juan Pablo.

Colegio de Bachilleres (1992). Reglamento del personal académico del Colegio de Bachilleres. México. Recuperado de http://www.cbachilleres.edu. $\mathrm{mx} / \mathrm{cbportal} / \mathrm{pdfs} / \mathrm{marco} / \mathrm{pdf} /$ interno/personal\%20academico/reg_pacad. 
pdfhttp://www.cbachilleres.edu.mx/cbportal/pdfs/marco/pdf/interno/ personal\%20academico/reg_pacad.pdf

De Ibarrola, M. (2015). La educación doctoral de los investigadores educativos: políticas y contextos nacionales y actores institucionales. En M. de Ibarrola y L. Anderson (2015). La formación de nuevos investigadores educativos. Diálogos y debates (pp. 67-103). México: ANUIES.

Diario Oficial de la Federación (2005). Decreto por el que se modifica el diverso que crea el Colegio de Bachilleres como organismo descentralizado del Estado, con personalidad jurídica, patrimonio propio y domicilio en la Ciudad de México, publicado el 26 de septiembre de 1973, México, DF.

Diario Oficial de la Federación (1973). Decreto de creación del Colegio de Bachilleres. Recuperado de http://bibliohistorico.juridicas.unam.mx/libros/5/2362/5.pdf

Dubar, C. (2000). La crisis de las identidades. La interpretación de una mutación. España: Ediciones Balleterra.

Fierro, C. (1999). Transformando la práctica docente. Una propuesta basada en la investigación-acción. México: Ediciones Paidós Ibérica, SA.

Evetts, J. (2003). Identidad, diversidad y segmentación profesional: el caso de la ingeniería. En Mariano Sánchez-Martínez, Juan Sáez y Lennart Svensson (coords.). Sociología de las profesiones. Pasado, presente y futuro. Murcia: Diego Marín Librero Editor.

Ezzy, D. (2002). Qualitative analysis: Practice and innovation. Crows Nest: Allen \& Unwin. Glaser, B. (1999) The future of grounded theory. Qualitative Health Research, vol. 9, núm. 6, pp. 836-845.

INEE (2015). Los docentes en México. Informe 2015. México.

Lanéelle, X. (2011). Troistransitions, deuxsituationsprofessionnelles, une rie: le cas des enseignants: intermittents. Recherchesenéducation, núm. 11, pp. 94-105.

Martuccelli, D. (2010). Los tres ejes de la identidad. En D. Gutiérrez. Epistemología de las identidades. Reflexiones en torno a la pluralidad (pp. 61-76). México: Universidad Nacional Autónoma de México.

Maccoby, E. E. \& Maccoby, N. (1954). The interview: A tool of social science. In G. Lindzey (ed.). Handbook of social psychology, vol. 1. Theory and method (pp. 449-487). Reading, MA: Addison-Wesley.

Martínez, M. (2017). De una identidad profesional a una identidad docente: profesores contratados por asignatura en el Colegio de Bachilleres de la Ciudad de México. Tesis de maestría, Cinvestav-IPN, México.

Merton, R. K. (1964). Teorías y estructuras sociales. México: Fondo de Cultura Económica (segunda reimpresión 1970).

Miles, M. B. \& Huberman, A. M. (1994). Qualitative data analysis: An expanded sourcebook. Thousand Oaks, CA: Sage Publications.

Monereo, C. (s.f.). La perspectiva dialógica en la construcción de la identidad humana a lo largo de la vida (manuscrito inédito).

SEP-COBACH (1973). Decreto de creación del Colegio de Bachilleres. Recuperado de http://www.cbachilleres.edu.mx/cbportal/pdfs/marco/pdf/interno/ atribuciones,\%20organizacion $\% 20 \mathrm{y} \% 20$ planeacion/dc_creacb.pdf 
Apéndice. Rasgos básicos de los profesores entrevistados

\begin{tabular}{|c|c|c|c|c|c|c|c|c|c|c|c|}
\hline Clave & Sexo & $\begin{array}{l}\text { Formación } \\
\text { profesional }\end{array}$ & Asignatura & $\begin{array}{c}\text { Horas } \\
\text { contra- } \\
\text { tado } \\
\text { a las } \\
\text { semana }\end{array}$ & $\begin{array}{l}\text { Grupos } \\
\text { que } \\
\text { atienda }\end{array}$ & $\begin{array}{l}\text { Semes- } \\
\text { tres } \\
\text { que } \\
\text { atiene }\end{array}$ & $\begin{array}{c}\text { Años } \\
\text { laborando } \\
\text { en el } \\
\text { COBACH }\end{array}$ & $\begin{array}{c}\text { Forma de } \\
\text { ingreso }\end{array}$ & $\begin{array}{l}\text { Turno } \\
\text { en que } \\
\text { labora }\end{array}$ & $\begin{array}{c}\text { Categoría } \\
\text { de contra- } \\
\text { tación }\end{array}$ & $\begin{array}{c}\text { Otro } \\
\text { empleo }\end{array}$ \\
\hline B1 & Femenino & $\begin{array}{l}\text { Licenciada en } \\
\text { Historia }\end{array}$ & $\begin{array}{l}\text { Introduc- } \\
\text { ción a las } \\
\text { Ciencias } \\
\text { Sociales }\end{array}$ & 27 & 6 & $2^{\circ}$ & 37 años & $\begin{array}{l}\text { Dejó pa- } \\
\text { peles en } \\
\text { dirección } \\
\text { general }\end{array}$ & Mixto & C1 & No \\
\hline $\mathrm{R} 2$ & Masculino & $\begin{array}{l}\text { Ingeniero en } \\
\text { Comunicacio- } \\
\text { nes Electró- } \\
\text { nicas }\end{array}$ & $\begin{array}{c}\text { Física } 1 \\
\text { Física } 2 \\
\text { Física } 3 \\
\text { TIC } 1 \\
\text { TIC } 2\end{array}$ & 28 & 6 & $\begin{array}{l}2^{\circ} \\
4^{\circ}\end{array}$ & 16 años & $\begin{array}{l}\text { Recomen- } \\
\text { dación de } \\
\text { una profe- } \\
\text { sora }\end{array}$ & $\begin{array}{l}\text { Vesper- } \\
\text { tino }\end{array}$ & $\mathrm{C} 2$ & $\begin{array}{l}\text { Docente } \\
\text { en el } \\
\text { Colegio de } \\
\text { Ciencias y } \\
\text { Humani- } \\
\text { dades }\end{array}$ \\
\hline F3 & Masculino & $\begin{array}{l}\text { Licenciado en } \\
\text { Administra- } \\
\text { ción }\end{array}$ & $\begin{array}{l}\text { Admisión y } \\
\text { empleo } \\
\text { Admisión y } \\
\text { empleo } 2 \\
\text { Introduc- } \\
\text { ción al } \\
\text { trabajo } \\
\text { Nociones } \\
\text { de } \\
\text { auditoría }\end{array}$ & 27 & 6 & $6^{\circ}$ & 14 años & $\begin{array}{l}\text { Por medio } \\
\text { de un } \\
\text { curso }\end{array}$ & Mixto & C3 & Grafólogo \\
\hline S4 & Femenino & $\begin{array}{l}\text { Químico } \\
\text { Fármaco- } \\
\text { Biólogo }\end{array}$ & $\begin{array}{l}\text { Química } 1 \\
\text { Química } 2 \\
\text { Química } 3\end{array}$ & 18 & 6 & $\begin{array}{l}2^{\circ} \\
4^{\circ} \\
6^{\circ}\end{array}$ & Seis meses & $\begin{array}{l}\text { Concurso } \\
\text { de oposi- } \\
\text { ción }\end{array}$ & Mixto & $\mathrm{C} 1$ & $\begin{array}{c}\text { Docente } \\
\text { en pre- } \\
\text { paratoria } \\
\text { particular }\end{array}$ \\
\hline JA5 & Masculino & $\begin{array}{l}\text { Dr. en Filo- } \\
\text { sofía }\end{array}$ & Filosofía & 3 & 1 & $6^{\circ}$ & $\begin{array}{l}\text { Mes y } \\
\text { medio }\end{array}$ & $\begin{array}{c}\text { Dejó sus } \\
\text { papeles } \\
\text { en un } \\
\text { plantel }\end{array}$ & $\begin{array}{c}\text { Matuti- } \\
\text { no }\end{array}$ & C1 & $\begin{array}{l}\text { Docente } \\
\text { en la Fa- } \\
\text { cultad de } \\
\text { Filosofía } \\
\text { y Letras } \\
\text { (UNAM) }\end{array}$ \\
\hline F6 & Masculino & $\begin{array}{l}\text { Ingeniero } \\
\text { mecánico }\end{array}$ & Física & 4 & 1 & $4^{\circ}$ & 16 años & $\begin{array}{l}\text { Por medio } \\
\text { de un } \\
\text { curso }\end{array}$ & $\begin{array}{c}\text { Matuti- } \\
\text { no }\end{array}$ & C1 & $\begin{array}{l}\text { Docente } \\
\text { de prepa- } \\
\text { ratoria de } \\
\text { la Ciudad } \\
\text { de México }\end{array}$ \\
\hline J7 & Masculino & $\begin{array}{l}\text { Lic. en Econo- } \\
\text { mía }\end{array}$ & $\begin{array}{c}\text { Estructura } \\
\text { socioeco- } \\
\text { nómica de } \\
\text { México } \\
\text { Administra- } \\
\text { ción }\end{array}$ & 7 & 2 & $\begin{array}{l}2^{\circ} \\
4^{\circ}\end{array}$ & 5 años & $\begin{array}{l}\text { Por } \\
\text { medio de } \\
\text { recomen- } \\
\text { dación }\end{array}$ & Mixto & C1 & $\begin{array}{l}\text { Docente } \\
\text { en otro } \\
\text { Colegio de } \\
\text { Bachille- } \\
\text { res }\end{array}$ \\
\hline R8 & Masculino & $\begin{array}{l}\text { Ingeniero } \\
\text { Químico } \\
\text { Industrial }\end{array}$ & $\begin{array}{l}\text { Matemáti- } \\
\text { cas }\end{array}$ & 5 & 1 & $6^{\circ}$ & 10 años & $\begin{array}{l}\text { Dejó sus } \\
\text { papeles } \\
\text { en un } \\
\text { plantel } \\
\text { del CO- } \\
\text { BACH }\end{array}$ & Mixto & C1 & $\begin{array}{l}\text { Adminis- } \\
\text { trativo en } \\
\text { un colegio }\end{array}$ \\
\hline
\end{tabular}




\begin{tabular}{|c|c|c|c|c|c|c|c|c|c|c|c|}
\hline X9 & Femenino & $\begin{array}{l}\text { Lic. en Litera- } \\
\text { tura Dramáti- } \\
\text { ca y Teatro }\end{array}$ & $\begin{array}{l}\text { Literatura } 1 \\
\text { Literatura } 2\end{array}$ & 28 & 6 & $\begin{array}{l}2^{\circ} \\
4^{\circ}\end{array}$ & 16 años & $\begin{array}{l}\text { Dejó sus } \\
\text { papeles } \\
\text { en la } \\
\text { Dirección } \\
\text { General } \\
\text { del CO- } \\
\text { BACH }\end{array}$ & Mixto & $\mathrm{C} 2$ & No \\
\hline R10 & Femenino & $\begin{array}{l}\text { Lic. en Perio- } \\
\text { dismo }\end{array}$ & $\begin{array}{l}\text { Lenguaje y } \\
\text { comunica- } \\
\text { ción } \\
\text { Ciencias de } \\
\text { la comuni- } \\
\text { cación }\end{array}$ & 28 & 7 & $\begin{array}{l}2^{\circ} \\
6^{\circ}\end{array}$ & 12 años & $\begin{array}{l}\text { Dejó sus } \\
\text { papeles } \\
\text { en la } \\
\text { Dirección } \\
\text { General }\end{array}$ & Mixto & C1 & No \\
\hline M11 & Femenino & $\begin{array}{l}\text { Ingeniera } \\
\text { Química } \\
\text { Petrolera }\end{array}$ & $\begin{array}{l}\text { Matemáti- } \\
\quad \text { cas } \\
\text { Química }\end{array}$ & 27 & 6 & $\begin{array}{l}2^{\circ} \\
4^{\circ} \\
6\end{array}$ & $\begin{array}{c}1 / 2 \\
\text { año }\end{array}$ & $\begin{array}{l}\text { Examen } \\
\text { de oposi- } \\
\text { ción }\end{array}$ & Mixto & $\mathrm{C} 1$ & No \\
\hline 12 & Femenino & $\begin{array}{c}\text { Medica } \\
\text { Veterinaria } \\
\text { Zootecnista }\end{array}$ & $\begin{array}{l}\text { Ecología- } \\
\text { logía }\end{array}$ & 15 & 5 & $6^{\circ}$ & $1 / 2$ año & $\begin{array}{l}\text { Examen } \\
\text { de oposi- } \\
\text { ción }\end{array}$ & $\begin{array}{l}\text { vesper- } \\
\text { tino }\end{array}$ & C1 & No \\
\hline
\end{tabular}

